\title{
Couette Flow of a Casson Fluid in an Inclined Composite Duct
}

\section{Manoj Kumar Uppuluri, S. Sreenadh, G. Gopi Krishna, A. N. S. Srinivas}

\begin{abstract}
Couette flow of a Casson fluid in an inclined composite duct comprising of porous and fluid layers of different thickness is investigated. A fully developed steady flow is assumed to be produced by applying a constant pressure gradient. The flow governing equations are described as per the standard literature. The boundary conditions at the porous and free fluid interface are formulated and the equations for fluid velocity within the porous layer and in the open channel are solved analytically. These flow quantities are studied for the effect of parameters such as porous layer thickness, volume fraction of fluid, viscosity parameter, drag parameter, angle of inclination, upper plate velocity and Casson fluid parameter. The results obtained are compared with standard literature and show good agreement. The study reveals that under prescribed conditions fluid flux in the porous layer is higher than that of in the open channel.
\end{abstract}

\begin{tabular}{|c|c|}
\hline & Nomenclature \\
\hline $\begin{array}{l}g \text { Gravitation. } \\
K \text { Drag coefficient. } \\
h_{1} \text { Height of free } \\
\text { flow region. } \\
h_{2} \text { Height of the } \\
\text { porous wall. } \\
q \text { Fluid velocity in } \\
\text { channel. } \\
\beta \text { Casson fluid } \\
\text { parameter. } \\
\delta \text { Viscous drag. }\end{array}$ & $\begin{array}{l}\mu_{a} \text { Apparent viscosity of the fluid } \\
\text { through the porous wall. } \\
\mu_{f} \text { Viscosity Coefficient. } \\
v \quad \text { Velocity of the fluid through the } \\
\text { porous layer. } \\
\phi \text { The volume fraction of thefluid. } \\
\theta \text { Angle of inclination. } \\
-\partial p / \partial x=G_{0} \text { Typical pressure } \\
\text { gradient. }\end{array}$ \\
\hline
\end{tabular}

\section{INTRODUCTION}

Flows through and past a porous material have applications in Engineering and Medicine. The rigging of crude oil and gasoline from natural reservoirs can be improved through principles of flows through porous medium. Potential applications are found in modelling of bio fluids such as blood. Many of these situations involve a composite duct constituted with a part of fluid layer and a part of saturated porous layer adjacent to it. Fluid flow through composite layers is also studied in perspective of many engineering applications such as porous insulation of ducts, fibrous and granular insulation and drying paper. Several researchers worked on composite layer flows. (Vafai and Kim[1], Kuznetsov[2], Alazami and Vafai[3])

Revised Manuscript Received on December 15, 2019.

V. Manoj Kumar Uppuluri, Division of Mathematics, Department of S \& H, Vignan's Foundation for Science Technology and Research, Vadlamudi, Guntur, A.P., India- 522213.

S. Sreenadh, Department of Mathematics, Sri Venkateswara University, Tirupati, A.P., India- 517502.

G. Gopi Krishna, Department of Mathematics, Marri Laxman Reddy Institute of Technology and Mangement,

Hyderabad, A.P., India- 500043

A. N. S. Srinivas, Department of Mathematics, School of Advanced Sciences, VIT, Vellore, 632 014, Tamilnadu, India
In modelling mammal joint lubrications and porous journal bearings the flow is considered as Couette flow through a composite duct. The boundary condition at the porous and free fluid interface play a key role in fluid mechanics of joint lubrications. Beavers and Joseph [4] proposed a slip velocity boundary condition at the porous and free fluid interface and verified its validation experimentally. Many researchers investigated the applicability and limitations of Darcy Law, DarcyBrinkman, Brinkman-Forchheimer models in formulating the boundary conditions at the solid boundary between a porous layer and a fluid ( Rudraiah [5], Neild [6], Vafai \& Kim [7]).

Hou et al., [8] in their study of boundary conditions at the cartilage-synovial fluid interface for joint lubrication derived meaningful boundary conditions at a fluid and permeable porous layer interface. Literature reveals that non-Newtonian fluid models are best to describe the behaviour of synovial fluid. Casson fluid model is such a non-Newtonian model widely used by researchers for modelling blood and other bio fluids.( Ahmed, Javed \& Sajid [9]; Akbarzadeh [10]; Rashidi , Yang, Bhatti \& Abbas [11]). Sreenadh et al.[12-15] studied some problems involving Casson fluid flow models.

In this perspective, Couette flow of a Casson fluid in an inclined composite duct partly filled with fluid and partly filled with porous material is investigated. The velocity of the fluid through porous layers and velocity of fluid in free flow region are calculated. These flow quantities are investigated for varying physical parameters and results are discussed using graphical representations.

\section{MATHEMATICAL FORMULATION}

In this study we consider fully developed steady Couette flow of a Casson fluid in an inclined composite duct partly filled with Casson fluid and partly filled with rigid porous material of heights $h_{1}$ and $h_{2}$ respectively. The walls of the channel are at $y=h_{1}$ and $y=-$ $h_{2}$, upper wall at $y=h_{1}$ is moving with a velocity $u_{0}$, as shown in the Figure - 1 below 


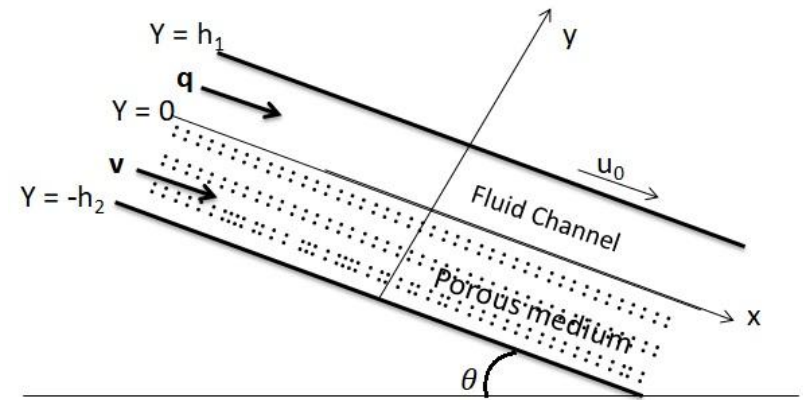

Figure 1: Physical model

The fluid velocity through the porous layers is $(v, 0,0)$, and the free flow velocity of the fluid is taken as $(q, 0,0)$. An axially directed flow is produced by applying a pressure gradient $-\frac{\partial p}{\partial x}=G_{0}$ on the channel.

As per the assumptions made above, the governing differential equations for the proposed flow are as follows $\mu_{a}\left(1+\frac{1}{\beta}\right) \frac{\partial^{2} v}{\partial y^{2}}-\phi \frac{\partial p}{\partial x}+\rho g \sin \theta-K v=0$ in $-h_{2} \leq y \leq$ $\mu_{f}\left(1+\frac{1}{\beta}\right) \frac{\partial^{2} q}{\partial y^{2}}-\frac{\partial p}{\partial x}+\rho g \sin \theta=0$ in $0 \leq y \leq h_{1}$

The boundary conditions are

$$
y=h_{1}: q=u_{0},
$$

$y=-h_{2}: v=0$,

$y=0: \quad \phi v=q, \phi \mu_{f} \frac{d q}{d y}=\mu_{a} \frac{d v}{d y}$

$$
y=h y^{*}, q=\frac{-h^{2} G_{0}}{\mu_{f}} q^{*}, v=\frac{-h^{2} G_{0}}{\mu_{f}} v^{*}, \eta=\frac{\phi^{2} \mu_{f}}{\mu_{a}}, N=\frac{\rho g}{G_{0}}, \varepsilon=
$$

where $\delta$ is a parameter that measures the fluid's viscous drag relative to that of the drag in the porous medium and $h$ $=h_{1}+h_{2}$. The above substitutions non-dimensionalizes the equations from (1) and (2) along with boundary conditions (3) and reduces them as follows (*s are neglected)

$$
\begin{aligned}
& \frac{d^{2} v}{d y^{2}}-\frac{\beta}{\delta^{2}(1+\beta)} v=-\frac{(N \sin \theta+\phi) \eta \beta}{\phi^{2}(1+\beta)} \\
& \frac{d^{2} q}{d y^{2}}+\frac{\beta(N \sin \theta+1)}{1+\beta}=0
\end{aligned}
$$

The resulting boundary conditions are

$$
\begin{aligned}
& y=\varepsilon: \quad q=u_{0} \text {, } \\
& y=\varepsilon-1: \quad v=0, \\
& \begin{array}{l}
y=0: \\
\leq 0
\end{array} \quad q=\phi v, \frac{d q}{d y}=\frac{\eta}{\phi} \frac{d v}{d y} .
\end{aligned}
$$

\section{SOLUTION FOR THE PROBLEM}

The coupled differential equations (4) - (5) are solved subject to the boundary conditions (6) analytically. The dimensionless fluid velocities of free flow and porous region flow are given by

$$
\begin{aligned}
& v(y)=c_{1} e^{\left(\frac{\sqrt{\beta}}{\delta \sqrt{1+\beta}}\right) y}+c_{1} e^{\left(\frac{-\sqrt{\beta}}{\delta \sqrt{1+\beta}}\right) y}+\frac{\eta \delta^{2}}{\phi^{2}}(\phi+N \sin \theta) \\
& q(y)=\frac{-(1+N \sin \theta) \beta}{1+\beta} \frac{y^{2}}{2}+c_{3} y+c_{4}
\end{aligned}
$$

Where

\section{NONDIMENSIONALIZATION OF THE FLOW QUANTITIES}

Consider the following substitutions

$$
c_{1}=\frac{\eta\left(\left(\frac{u_{0}}{\phi}+\frac{\varepsilon^{2}(1+N \sin \theta) \beta}{2 \phi(1+\beta)}\right) e^{\frac{(1-\varepsilon) \sqrt{\beta}}{\delta \sqrt{1+\beta}}}+\frac{\delta^{2} \eta(\phi+N \sin \theta)}{\phi^{2}}\right)-\frac{\varepsilon \sqrt{\beta}}{\delta \sqrt{1+\beta}}\left(\frac{\delta^{2} \eta(\phi+N \sin \theta)}{\phi^{2}}\right)-\left(\frac{\delta^{2} \eta^{2}(\phi+N \sin \theta)}{\phi^{2}}\right) e^{\frac{(1-\varepsilon) \sqrt{\beta}}{\delta \sqrt{1+\beta}}}}{2 \eta \sinh \left(\frac{(1-\varepsilon) \sqrt{\beta}}{\delta \sqrt{1+\beta}}\right)+2 \frac{\varepsilon \sqrt{\beta}}{\delta \sqrt{1+\beta}} \cosh \left(\frac{(1-\varepsilon) \sqrt{\beta}}{\delta \sqrt{1+\beta}}\right)}
$$

$c_{2}=-\frac{(\phi+N \sin \theta) \delta^{2} \eta}{\phi^{2}} e^{\frac{-\sqrt{\beta}}{\delta \sqrt{1+\beta}}}-c_{1} e^{\frac{-2 \sqrt{\beta}}{\delta \sqrt{1+\beta}}}$

$c_{3}=\frac{\phi}{\eta} \frac{\sqrt{\beta}}{\delta \sqrt{1+\beta}}\left(c_{1}-c_{2}\right)$,

$c_{4}=\phi\left(c_{1}+c_{2}+\frac{(\phi+N \sin \theta) \delta^{2} \eta}{\phi^{2}}\right)$

\section{RESULTS DESCRIPTION}

Couette flow of a Casson fluid in an inclined composite duct comprising of porous and fluid layers of different thickness is investigated. Analytical solutions for the velocity of fluid through porous layer, the free flow velocity of the fluid are obtained. These flow quantities are investigated for different parameters such as Casson fluid perameter $\beta$, volume fraction of the fluid $\phi$, drag coefficient $\delta$, 
thickness of the porous layer $\mathcal{E}$, upper plate velocity $u_{0}$, viscosity parameter $\eta$, pressure gradient parameter $N$ and angle of inclination $\theta$. Throughout this study numerical calculations are carried out by assuming the following values: $N=1, \phi=0.6, \delta=\frac{1}{\sqrt{2}}$,

$\eta=0.36, \varepsilon=0.5, \beta=-2, u_{0}=0.5 \quad$ and $\theta=\pi / 3$

unless otherwise stated.

Equation (7) and (8) gives the relationship between fluid velocity in porous media $v$ with $y$ and free flow region velocity $q$ with $y$, for varying values of the parameters $\delta, \phi, \eta, \theta, \beta, u_{o}$ and $N$, which are represented graphically through figures 2 to 8 respectively. Figure 2 depicts that enhancing the values of the drag parameter $\delta$ enhances the velocity of the fluid in porous region as well as in free flow region. Figure 3 depicts that the flow velocity in both layers is increasing with decreasing values of $\phi$. From figure 4 it can be interpreted that with an increase in the viscosity parameter $\eta$, fluid velocity in both layers is increasing. As angle of inclination $\theta$ increases, the buoyancy forces also increases, which results an increases in the flow velocity in both the layers, as shown in Figure 5. Flow velocity in both the porous and fluid only regions is increasing with increasing values of Casson parameter $\beta$ which is depicted in figure 6. Figure 7 shows that the flow velocity in porous as well as fluid only regions increases by increasing the upper plate velocity $u_{0}$. Figure 8 shows that flow velocity in porous as well as fluid only regions is increasing with increasing values of pressure gradient parameter $N$.

Fig.9 depicts the fluid flux variations for different values of $\delta$ varying with different

values of $u_{o}$ when $\phi=1, \varepsilon=0.5, \eta=1, \beta=-2, \theta=0$ and $N=1$. The values taken here for the parameters $\delta, \phi, \varepsilon, \eta, \beta, \theta$ reduces the present problem into Couette flow of a viscous fluid over a permeable porous bed and the results obtained in this case matches with that of

Hou et.al., [8]. For upper plate velocity $u_{o}=0$ we can observe a boundary layer in porous layer for smaller values of $\delta$.

Fig.10(A), (B) depicts fluid flux variations for different values of $\varepsilon$ when $\phi=1, \eta=1, \beta=-2, \delta=1, \theta=\frac{\pi}{3}$, $u_{o}=0$ and $N=1$. When $\varepsilon=0$, height of the free flow region becomes zero, i.e. the channel is entirely filled with porous medium and when $\varepsilon=1$, height of the porous layer becomes zero, i.e. the channel has no porous medium. Fig. 10(A) depicts the fluid fluxes for the above two special cases and we can observe that fluid flux through the channel that is completely filled with porous medium is less than the fluid flux through channel without porous medium.

Fig. 10(B) depicts the fluid flux variations for different values of $\varepsilon$ when $\phi=1, \eta=1, \beta=-2, \delta=1, \theta=\frac{\pi}{3}$, $u_{o}=0$ and $N=1$. It should be noted that when $\varepsilon=0.25$ the

fluid flux through porous medium is more than the fluid flux through free flow region.

\section{CONCLUSIONS}

Couette flow of a Casson fluid through an inclined composite duct with porous and clear fluid layers of different thickness is investigated. The expressions for fluid velocities in both the layers of the duct are obtained analytically. These flow quantities are investigated for varying physical parameters and results are discussed using graphical representations. The following are some of the important observations:

1. It is observed that velocities of the fluid in both regions of the duct increases by increasing the value of the drag parameter $\delta$, viscosity parameter $\eta$, angle of inclination $\theta$, Casson fluid parameter $\beta$, upper plate velocity $u_{o}$ and pressure gradient parameter $N$.

2. The flow velocity in the porous media also in free flow region decreases with increase of volume fraction $\phi$.

3. It is noticed that a boundary layer flow exists in porous region for too small values of the

drag parameter $\delta$.

4. When free flow region height is too small and value of drag parameter $\delta$ is almost nearer to unity, the fluid flux in porous region is more than the fluid flux in free flow region.

\section{REFERENCES}

1. Vafai, K., Kim, S.J.:Fluid mechanics of interface region between a porous medium and a fluid layer- an exact solution. Int. J. Heat Mass Transfer 11, 254-256 (1990)

2. Kuznetsov, A.V.:Analytical investigation of Couette flow in a composite channel Partially filled with a porous medium and partially with a clear fluid. Int. J. Heat Mass Transfer 41, 2556-2560, (1998)

3. Alazami, M., Vafai, K. : Analysis of fluid flow and heat transfer interfacial conditions

4. between a porous medium and a porous layer. Int. J. Heat Mass Transfer 44, 1735-1749 (2001)

5. Beavers, G. S., Joseph, D.D.: Boundary conditions at naturally permeable wall. J. Fluid Mech. 13, 197-207 (1967)

6. Rudraiah, N.: Forced convection in a parallel plate channel partially filled with a porous material. ASME J. Heat Transfer 107, 331-332 (1985)

7. Neild, D.A.: The limitations of the Brikman-Forchheimer equation in modeling flow in a saturated porous medium and at an interface. Int. J. Heat Mass Transfer 12, 269-272 (1991)

8. Vafai, K., Kim, S.J.: On the limitation of the Brinkman Forchheimerextended Darcy equation. Int. J. Heat Mass Transfer 16, 11-15 (1995)

9. J. S. Hou, M. H. Holmes, W. M. Lai and V. C. Mow, Boundary conditions at the cartilage -synovial fluid interface for joint lubrication and theoretical verifications. J. Biomech. Engng., 111, 7887 (1989).

10. Ahmed BI, Javed TA, Sajid MU. Peristaltic transport of blood in terms of Casson fluid model through a tube under impact of magnetic field for moderate Reynolds number. J Qual Meas Anal. 2018;14(1):101-13. Akbarzadeh P. Pulsatile magneto-hydrodynamic blood flows through porous blood vessels using a third grade nonNewtonian fluids model. Computer methods and programs in biomedicine. 2016 Apr 1;126:3-19.

11. Rashidi MM, Yang Z, Bhatti MM, Abbas MA. Heat and mass transfer analysis on MHD blood flow of Casson fluid model due to peristaltic wave. Therm. Sci. 2018 Jan 1;22: 2439-48. 
12. Sreenadh S, Pallavi AR, Satyanarayana BH. Flow of a Casson fluid through an inclined tube of non-uniform cross section with multiple stenoses. Advances in Applied Science Research, 2(5), 340-349 (2011)

13. Selvi, P.D., Sreenadh, S., Kesava Reddy, E., Pulsatile flow of a Casson fluid between permeable beds, International Journal of Mechanical Engineering and Technology, Volume 9, Issue 8, August 2018, pp. 589-600. (with P.D. Selvi and E. Kesava Reddy)

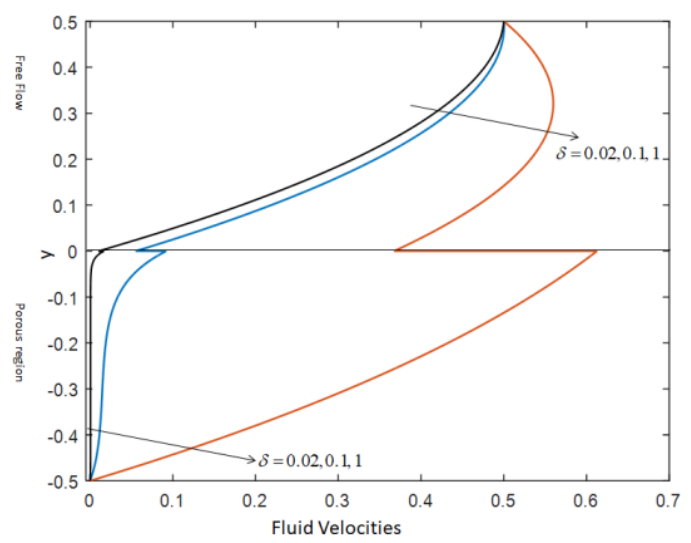

Fig. 2 Velocity profiles for varying values of $\delta$ with $u_{0}=0.5$

$N=1, \phi=0.6, \varepsilon=0.5, \eta=0.36$, and $\theta=\pi / 3 \beta=-2$,

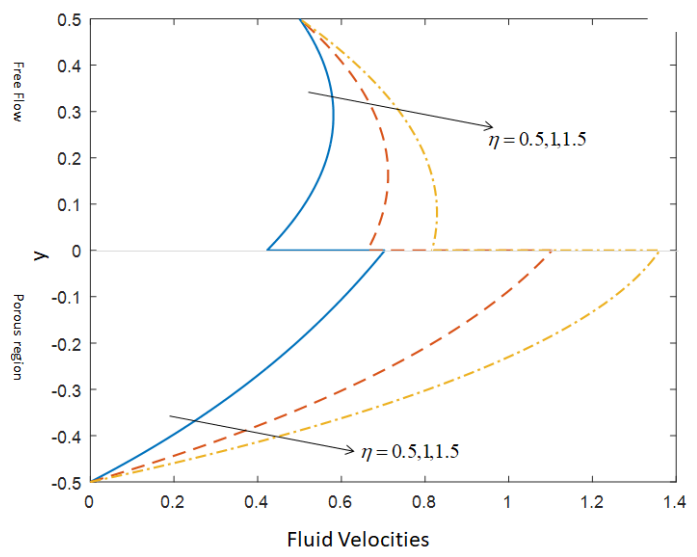

Fig. 4 Velocity profiles for varying values of $\eta$ with $u_{0}=0.5$

$N=1, \phi=0.6, \varepsilon=0.5, \delta=\frac{1}{\sqrt{2}}$, and $\theta=\pi / 3 \beta=-2$,

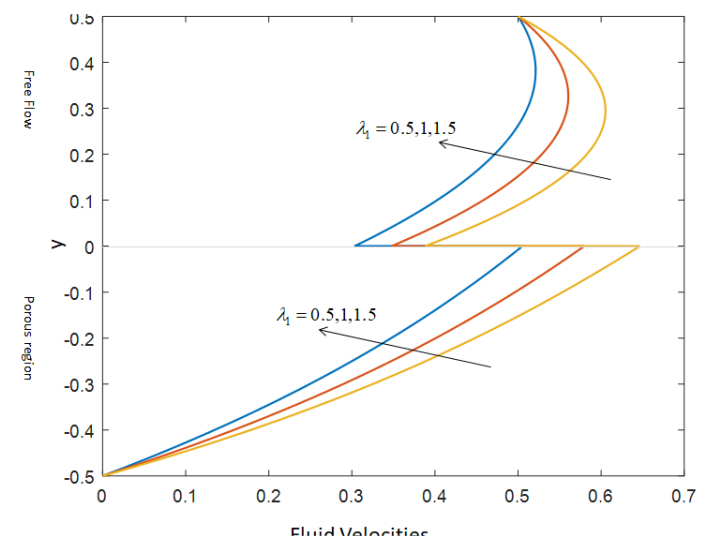

Fig. 6 Velocity profiles for varying values of $\lambda_{1}$ with $u_{0}=0.5$

$N=1, \phi=0.6, \varepsilon=0.5, \delta=\frac{1}{\sqrt{2}}, \eta=0.36$, and $\theta=\pi / 3$
14. S. Sreenadh, G. Gopi Krishna, V. Manoj Kumar Uppuluri and A.N.S.Srinivas. Viscous fluid flow in an inclined channel with deformable porous medium. International Journal of Mechanical Engineering and Technology (IJMET), Volume 9, Issue 1, January 2018, pp. 970-979

15. Vajravelu K, Sreenadh S, Devaki P, Prasad KV. Peristaltic pumping of a Casson fluid in an elastic tube. Journal of Applied Fluid Mechanics. 2016 Jan 1;9(4):1897-905.

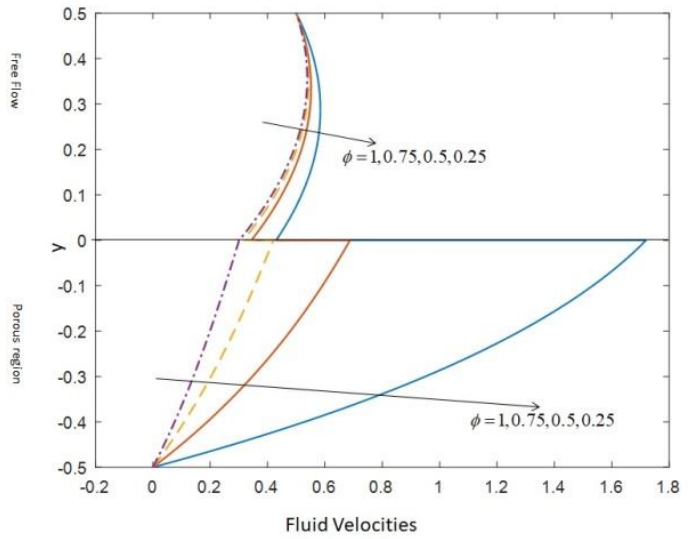

Fig. 3 Velocity profiles for varying values of $\phi$ with $u_{0}=0.5$ $N=1, \varepsilon=0.5, \delta=\frac{1}{\sqrt{2}}, \eta=0.36$, and $\theta=\pi / 3, \beta=-2$,

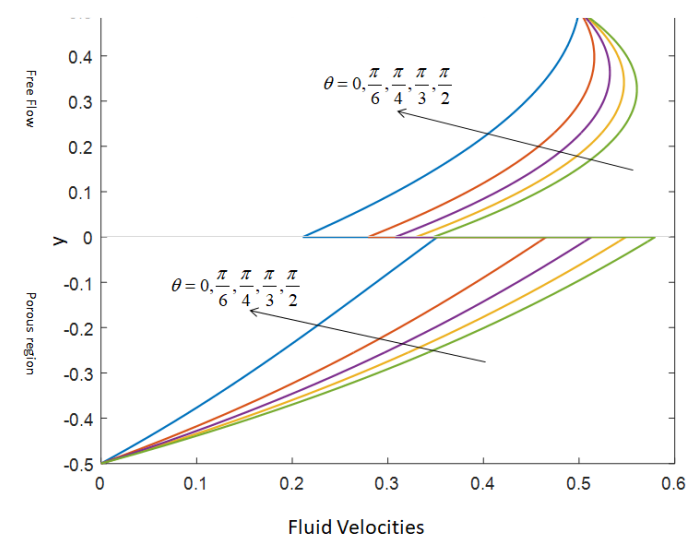

Fig. 5 Velocity profiles for varying values of $\theta$ with $u_{0}=0.5$ $N=1, \phi=0.6, \varepsilon=0.5, \delta=\frac{1}{\sqrt{2}}, \eta=0.36$, and $\beta=-2$,

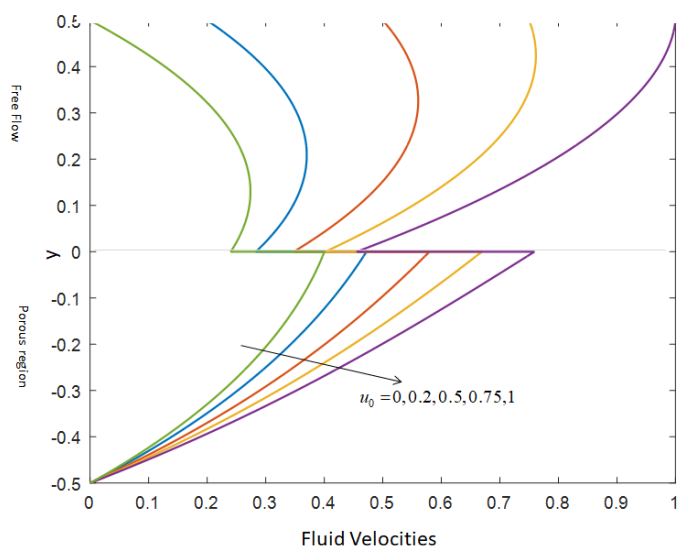

Fig. 7 Velocity profiles for varying values of $u_{0}$ with $N=1$, $\phi=0.6, \varepsilon=0.5, \delta=\frac{1}{\sqrt{2}}, \eta=0.36, \beta=-2$, and $\theta=\pi / 3$ 


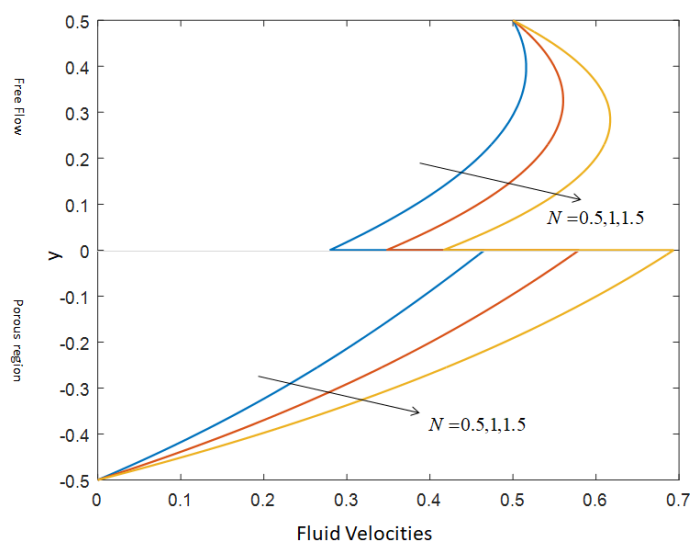

Fig. 8 Velocity profiles for varying values of $N$ with $u_{0}=0.5$ $\phi=0.6, \varepsilon=0.5, \delta=\frac{1}{\sqrt{2}}, \eta=0.36, \beta=-2$, and $\theta=\pi / 3$

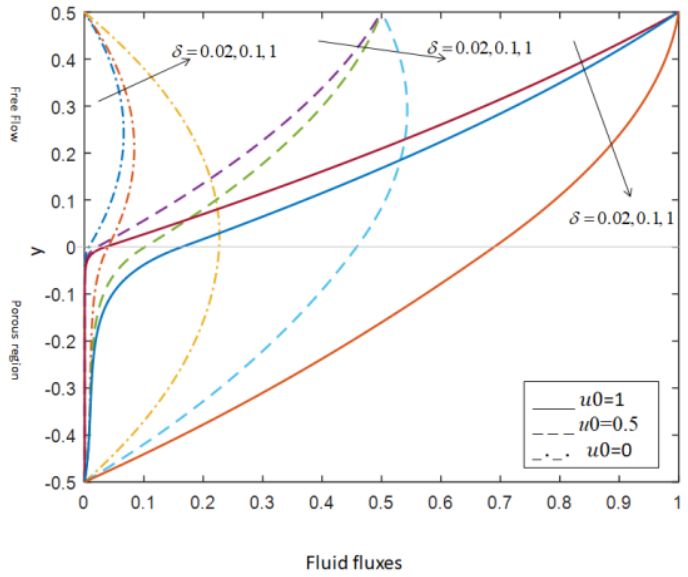

Fig. 9 Fluid flux profiles for various values of $\delta$ and $u_{0}$ with $\phi=1, \varepsilon=0.5, \eta=1, \beta=-2 \quad \theta=0$ and $N=1$

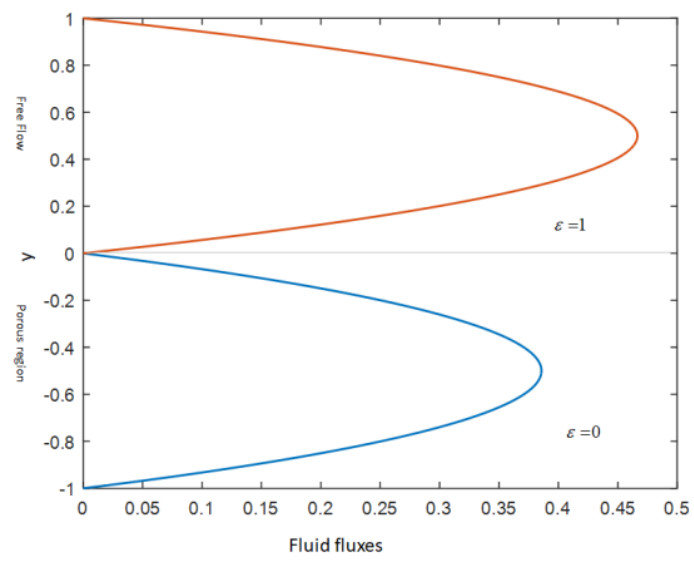

Fig. 10(A) Fluid flux profiles for various values of $\varepsilon$ with $\phi=1, \delta=1, \beta=-2, \eta=1, \theta=\frac{\pi}{3}, u_{0}=0$ and $N=1$

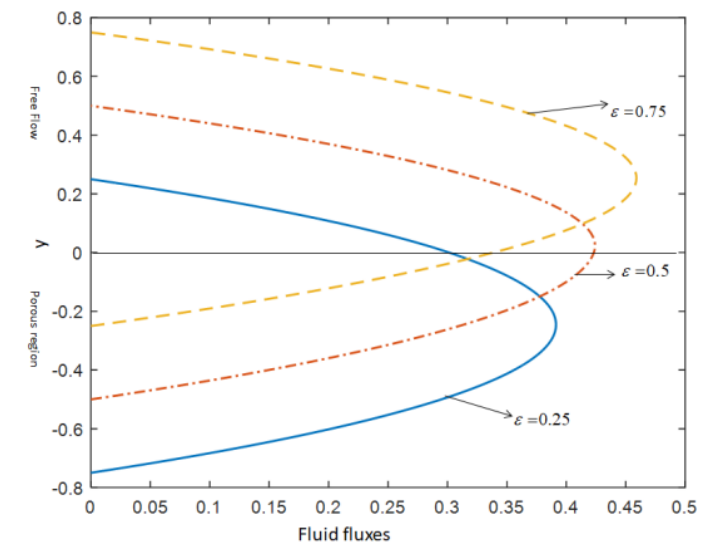

Fig. 10(B) Fluid flux profiles for various values of $\varepsilon$ with $\phi=1, \delta=1, \beta=-2, \eta=1, \theta=\frac{\pi}{3}, u_{0}=0$ and $N=1$ 